\title{
ASEAN luego de la tercera reunión cumbre
}

En medio de los mayores preparativos de seguridad imaginables el 14 y 15 de diciembre de 1987 se llevó a cabo en Manila la tercera reunión cumbre de los jefes de gobierno de la Asociación de Naciores de Sudeste Asiático (ASEAN) ${ }^{2}$. Hasta inmediatamente antes del inicio de la conferencia no era todavía seguro si la cumbre habría o no de reunirse, ya que la situación política en las Filipinas, extremadamente tensa, no era precisamente atrayente para el resto de los estados miembros de AsEAN. Recién después de que el Primer Ministro de Singapur Lee Kuan Yew y el Presidente de Indonesia Suharto se pusieran definitivamente de acuerdo sobre la realización de la conferencia en la Isla Batam (Indonesia) el 29 de noviembre, se despejó el camino para el encuentro en la capital filipina planeado ya largo tiempo atrás ${ }^{3}$. Lee Kuan Yew se refirió a la situación poIf́tica problemática en la que se encontraban en su discurso de apertura frente a la anfitriona, Corazón Aquino, con su característica franqueza: "Señora Presidente, si los jefes de gob:erno de ASEAN se dejaran guiar por sus servicios de seguridad, no estarían en Manila"4.

En las páginas que siguen no se ofrecerá primordialmente un análisis de la conferencia en cuanto tal, para lo cual el material disponible hasta el momento no sería suficiente ${ }^{5}$. Se trata más bien de

"Una primera versión de Ias reflexiones siguientes apareció bajo el titulo de "ASEAN am Vorabend der dritten Gipfelkonfernz" el 25 de noviembre de 1987, en Europa-Archiv, No.s 22-42, pp. 655-664. La versión actual ha sido modificada $\mathrm{y}$ ampliada en algunos puntos fundamentales, Iuego de que la cumbre de Manila sc ha llevado a cabo efectivamente.

-Cfr. Norbert Wagner, Drittes ASEAN-Gipfeltreffen in Manila, Singapur (comentario extenso del 16 de diciembre de 19S7) y también Rodney Tasker, "Leaders turn out to support Aquino despite risk. 18-minute solidarity", Far Eastern Economic Review, 24 de diciembre de 1987, pp. 8-9.

sCfr. Cheong Yip Seng y Yang Razali Kassim, "Summit Lee-Suharto talks", en: The Straits Times (Singapur), Iunes 30 de noviembre de 1987.

'Palabras de apertura del Primer Ministro Lee Kuan Yew, de la República de Singapur, en el tercer ASEAN Summit, 14 de diciembre de 1987, (mimeo).

ธPor el momento consiste prácticamente sólo en noticias de periódicos y breves reportajes de la prensa internacional. 
una valoración de ASEAN en general, con lo que la tercera reunión cumbre no e: nada más ni nada menos que una ocasión para un balance provisional. En el año de la cumbre de Manila la Asociación de Naciones del Sudeste Asiático cumplió veinte años. ¿Qué se ha logxado, dónde residen los problemas, cómo se continuará en el futuro? Y sobre todo: ttiene la organización una concepción clara y coherente de objetivos como base?

\section{La fundación}

ASEAN tiene una prehistoria algo larga y complicada que, en forma un poco simplificada, puede resumirse del siguiente modo: desde fines de la década del 50 , cuando - a excepción de Tailandia- en ninguno de lo; futuros países miembros el proceso de "nation-buiilding" había avanzado más allá de los primeros arranques, surgió la amplia necesidad de cooperar económica, cultural y técnicamente, por lo menos en forma suficiente como para activar el proceso de desarrollo propio sin resultar precipitadamente víctima de los intereses extranjero". EI "Esp'ritu de Bandung", con su filosofía de distanciamiento de toda tutela imperialista, constituyó un elemento de motivación, del mismo modo que "era inconfundible cierto acento

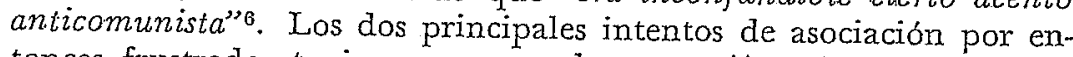
tonces frustrados tuvieron por nombre: ASA (Asrociation of Southeast Asia) y MAPHIIndo (denominación formada a partir de las letras iniciales de Ios paíse: miembros: Malasia, Philipinas e Indonesia). El 8 de agosto de 1967 los ministros del exterior de Indonesia, Malasia, Filipinas, Singapur y Tailandia, firmaron un breve documento que ingresó a la historia con el nombre de "Declaración de Bangkok" y que ha de ser visto como la carta de fundación de ASEAN.

ASEAN fue creada como una organización intergubernamental relativamente laxa "con el objetivo primordial de fortalecer a los estados miembros como unidades nacionales"7. Cualquier definición mán precisa de ASEAN que se oriente en forma más clara a objetivo; políticos, económicos o de cualquier otro tipo, hace perder de vista, en su unilateralidad, el hecho de que la Asociación de Naciones del Sudeste Asiático surgió primordialmente bajo el signo de nation building. Tal objetivo no se ha alterado tampoco en la actualidad. Las expectativas de la Asociación se discuten también en el Sudeste Asiático siempre en el contexto de un marco internacional capaz de

oWaIter $\mathrm{X}$. Rust, ASEAN Regionale Zusammenarbeit im Schatten der Grossmächte (Frankfurt, Berna, New York: Peter Lang, 1985), p. I6.

TMuthiah Alagappa, "ASEAN institutional framework and modus operandi: Recommandations for change", en: Noordin Sopiee, Ghew Lay See, Lim Siang Tin (eds.), ASEAN at the Crossroads: Obstacles, Options \& Opportunities in Economic Co-operation (Kuala-Lumpur: Institut of Strategic and International Studies), pp. 183-230; cit. p. 191. 
po:ibilitarlo y/o impedirlo. "National and regional resilience" es un doble concepto clave que aparece en casi todos los documentos y comunicados oficiales de la comunidad. La palabra resilience (difícil de traducir y que indica la capacidad que tiene un resorte de ceder en forma elástica y luego volver a retraerse a su forma original) ha sido introducida en la discusión programática interna del ASEAN por parte de Indonesia. National resilience designa "la capacidad de un pais de efectuar los cambios necesarios para el progreso social y económico y, al mismo tiempo, de encarar todas las amenazas externas preservando su identidad nacional esencial"s. No se trata en principio en la Confederación ASEAN -y ésa es una diferencia fundamental con respecto a los esfuerzos de cooperación e integración de Europa Occidental y América Latina- de una cooperación interestatal que tarde o temprano vaya a resultar en formas "superiores" o supranac:onales de cooperación que, por lo mismo, impliquen una renuncia parcial de soberanía. En primer plano se encuentra más bien la idea de hallar viabilidad y aceptación externa por medio de una cooperación interestatal en la subregión concebida en forma pragmática (= regional resilience) que asegure, en cambio, la capacidad de desarrollo y la situación de los países m embros.

Precisamente en el paso de 1987 a 1988 vale la pena examinar lo logrado por ASEAN hasta el momento, sobre todo en vistas a que en la conferencia cumbre de los jefes de Estado y de Gobierno en Manila se ha puesto en marcha una amplia discusión de reforma que en último término habría de servir para proporcionar a la comunidad una existencia exitosa en las próximas décadas.

\section{Cooperación en ASEAN: el aspecto politico}

En la Comunidad se parte de la idea de que el ingreso de otros paises que pertenezcan claramente a la región del Sudeste Asiático es, en principio, libre. En el caso de que, por ejemplo, en algún día lejano, se produjera un cambio de gobierno fundamental en Birmania y en los tres estados de Indochina, la asociación de esas naciones no habría de ser excluida a priori. Por el momento, sin embargo, la cuestión de la ampliación de la asociación se maneja más bien con cuidado y en forma reticente. De todos modos, el 7 de enero de 1984 el sultanato de Brunei pudo ingresar a la agrupación que originariamente contaba con cinco países. Papua-Nueva Guinea tiene desde hace un tiempo un status de observador en la ASEAN. En ese aspecto en la cumbre de Manila se produjo un claro avance en cuanto a una futura asociación plena al haberse admitido que ese

${ }^{8}$ Presidente Suharto, citado por David Irvine, "Making Haste Less Slowly: ASEAN from 1975", en: Alison Broinowski (ed.), Understanding ASEAN (London and Basingstoke: Macmillan, 1982), pp. 37-69; cit. p. 40. 
pafs se integre en un plazo previsible al "Treaty of Amisty and Cooperation in Southeast Asia", firmado por los gobiernos de Ia ASEAN en 1976. La extremá cautela que ha reinado hasta ahora en la cuestión de la asociación no es del todo infundada: no se quiere poner en juego lo alcanzado hasta el momento a causa de la expansión.

En el Tercer Mundo no hay ninguna otra agrupación que, como la ASEAN, haya logrado un diálogo regutar a nivel gubernamental con algunos de los actores de más alta posición de nuestro tiempo (Japón, Estados Unidos, la Comunidad Europea, Ganadá, Australia, Nueva Zelandia). Entre tanto el grupo ASEAN es respetado incluso por las potencias comunistas establecidas en la región. En ese sentido resulta ilustrativo el mensaje de salutación dirigido a la cumbre de Manila por el secretario general Gorbachov'. El programa regional de pacificación zopfan (Zone of Peace, Freedom and Neutrality Declaration) anunciado el 27 de noviembre de 1971 en Kuala Lumpur, Iuego de controversias iniciales, ha sido reconocido, incluso por sus enemigos originarios, como un concepto formativo concebible para la convivencia interestatal en el Sudeste Asiático.

A excepción del Golfo Pérsico no debe haber ninguna otra zona geopolftica en la que, como en el Sudeste Asiático, concurran tanto los intereses de las dos superpotencias y de las potencias regionales, como también las expectativas de Europa Occidental, AustraLia y IJueva Zelandia y, por lo mismo, entren parcialmente en coalición, tanto en lo estratégico como en lo concreto.

A través de su modo de comportarse, la Asociación ha sabido presentarse, en el juego de los intereses cruzados de terceros, como un subsistema que actúa en forma independiente en el campo de las fuerzas internacionales. Aquí han de recordarse, junto con la ZOPEAN y los programas de diálogo transformados desde hace tiempo en una rutina natural, las votaciones sobre la cuestión de Camboya en $\mathrm{I}_{a}$ onv, que a lo largo de muchos años se fueron articulando en forma cada vez más favorable a la posición de la ASEAN ${ }^{10}$. La Comunidad logró, sobre todo, transmitir hacia afuera una imagen de Ia región que le permitió hacerse juzgar según la base de un "balance de poder" interpretado en forma flexible y entrar en el cálculo de Ios intereses externos sin que internamente surja el sentimiento de un fatalismo resignado.

Se imponen c:ertos paralelismos con Europa Occidental: se trata de no ser sólo zona de influencia sino también de actuar como socio activo en el contexto internacional; Ios gobiernos de los estados

'Wagner, op. cit., p. 9.

${ }^{10} \mathrm{El}$ resultado de Ia rotación de la Asamblea General de la onv del 21 de octubre de 1986 se presenta ast: en favor de la resolución de la ASEAN sobre Camboya votaron 115 gobiernos, 21 en contra y 16 se abstuvieron. Todavia en 1979 el resultado era 91:21:29 (Fuente: ASEAN Newsletter, No 17, 1986, 6). 
de Ia ASEAN han estado igualmente dispuestos a aportar acreditadas relaciones internacionales propias (generalmente bilaterales) en beneficio de una diplomacia que trascienda lo nacional yique redunde en beneficio de la Comunidad. En este sentido su aceptación internacional es, con seguridad, uno de los mayores éxitos de la Asociación.

Cuánto se ha recorrido durante veinte años en esa dirección se aprecia, entre otras cosas, en el hecho de que hasta entrada la dé cada del seterita los Estados Unidos consideraban despréciativamen te a la ASEAN con "desinterés benévolo" en el mejor de los casos"1. A partir de 1977 ya no existe sólo un diálogo regular Estados UnidosASEAN. A comienzos del verano de 1986 el presidente Reagan se reunió en Bali con los seis ministros del exterior de la Comunidad.

Como ya se indicó, también para la Unión Soviética, para la República Popular China e incluso para Vietnam pueden anunciarse cambios que no deben pasar desapercibidos en lo que hace a su posición frente a Ia ASEAN, si bien los estados comunistas consideran a Ia ASEAN más como una realidad que hay que aceptar que como un socio internacional deseado.

En el balance de éxitos políticos cuenta además el hecho que, luego de dificultades iniciales en relación con una "renuncia fundamental a la violencia, es decir, al empleo de la violencia como medio para solucionar conflictos en el caso de intereses contradictorios entre los estadios"12, los países de la ASEAN se volvieron hacia una próspera relación con buena disposición al diálogo. Entretanto algunas tensiones pertenecen ya al pasado (por ejemplo la antigua posición "confrontasi" de Indonesia frente a Malasia o el conflicto de límites entre Malasia y Tailandia), otras no han sido todavía del todo dejadas de Iado (el conflicto de Saba entre Malasia y las Filipinas y los roces entre Singapur y Malasia condicionados en última inștancia por factores étnicos) y, finalmente, otras, por el contrario, volverán una y otra vez a recrudecer (disputas por las zonas de pes$\mathrm{ca}_{\mathrm{s} .}$ minorías religiosas o plataformas continentales). En todo caso es "sorprendente la renuncia interna de la Comunidad a la"utilización de violencia politico-militar para la solución de conflictos, si se recuerda que todavia en 1966 tres de los seis estados de la ASEAN (Indonesia, Malasia y Filipinas) se hallaban en un estado de tensión cuasi bélicio entre si'13.

دEva-Maria Masyk, Die Aussenpolitik der Vereinigten Staaten von Amerike gegenüber ASEAN unter der Reagan-Administration (München: 1986), p. 272.

${ }^{12}$ Rudiger Machetzki, "ASEAN-Leistunsprofile, Märkte und Kooperations-aussichten", separata de Südostasien Aktuell, julio de 1984, pp. 333.355; cit. p. 334,

${ }^{2 D e l}$ mismo, "ASEAN: Entwicklungsmodell mit Hindernissen", en: Aus Politik un Zeitgeschichte, 33/84, 18 de agosto de 1984, pp. 3-16; cit. p. 3. 
Si a partir de allí Rüdiger Machetzki14 subraya con mucha razón "la certeza de la estabilidad politica" como el "resultado de mayor importancia" de la ASEAN, habrá de tenerse también en cuenta su amplio sistema de consulta y decisión. Esto se aplica, en primer lugar, al nivel gubernamental pero también, en medida creciente; al nivel transnacional de las Organizaciones No Gubernamentales (ONGS). No se trata en su mayoría del cumplimiento práctico y organizativo de un gran plan, cosa que no puede esperarse porque la base jurídica de la ASEAN consiste en dos "Declaraciones" ("Bangkok" 1967.y "ASEAN Concond" 1976) relativamente breves y vagas y no en un documento contractual preciso, como es usual en otras regiones para similares esquemas de cooperación. Como se sabe (si.se dejan de lado impulsos que no carecen de importancia, como el establecimiento de una conferencia de ministros de economía y la instalación de una modesta secretaría en Yakarta, ambos recién a partir de 1976), la organización ha crecido en su estructura en forma pragmática y: según una lógica que podría ser calificada de increrientalismo funcional. La cooperación se amplía en la medida en que se plantean nuevas exigencias y "situaciones". En la actualidad debe haber todavía algún ministro que no se encuentre regularmente con sus colegas de los restantes gobiernos de los estados miembros en las conferencias de la ASEAN, lo que mutatis mutandis vale también para los niveles inferiores de las administraciones nacionales. Las agendas oficiales de la ASEAN están en todo caso atiborradas de citas y correspondientes órdenes del día ${ }^{15}$. En forma paralela ha surgido un tejido relativamente denso de actores transnacionales de - Ia ASEAN. En casos particulares el paso de los niveles gubernamentales a los no gubernamentales puede ser fluido. De ese modo las cámaras de industria y comercio, los bancos, las líneas de navegación, como así sectores de la industria, sindicalistas y parlámentarios y también asociaciones de jóvenes y deportistas van llevando a cabo lo que el premier de Singapur Lee Kuan Yew quiso dar a entender con el slogan: "Think $A S E A N$ ".

No cabe duda que tras este enorme éxito político está el intento de alcanzar comunicación, acuerdo y medidas conjuntas por medio de conexiones entre actores estatales, paraestatales y sociales, y así reducir, por medio de un diálogo de buena vecindad, Ias múltiples divergencias étnicas, religiosas, geopolítico-estratégicas, ideológicopolíticas, etc. entre los miembros de la ASEAN $\dot{y}$ limitar actitudes individuales demasiado egoístas de los estados nacionales. Esta múltiple red de interacciones no suprime la capacidad nacional de decisión ni las polfticas nacionales de desarrollo, de tránsito; exteriores,

MIbid.

${ }^{25}$ Cfr. ASEAN Secretariat, Annual Report of the ASEAN Standing .Committe 1986-1987 (Yacarta: 1987). 
económicas y sociales, pero ofrece la oportunidad de una polftica sectorial armonizada mulilateralmente. Si por un lado los gobiernos de la ASEAN observan con mucho cuidado que por medio del diálogo político intra-ASEAN no hayan de producirse pérdidas reconocibles de soberanía, por otro, formas manifiestas e intenciones declaradas de cooperación se extienden incluso en ámbitos políticos evidentemente sensibles: por ejemplo en una cooperación parcial en cuestiones de defensa o en un paso cauteloso hacia la equiparación de cuestiones legales ${ }^{16}$.

El hecho en sí mismo extraño que los jefes de estado y de gobierno hayan viajado a Manila, a pesar del consejo expreso de sus funcionarios de seguridad, con la intención públicamente declarada de contribuir de esa manera a la estabilización de la situación política en las Filipinas tno es acaso, mucho más que un simple gesto de solidaridad colegiada, la responsabilidad por la estabilidad política regional? Ésta se demuestra, incluso, bajo un riesgo personal, allí donde por medio de la acción (en caso contrario, de la omisión) se afecta la estabilidad interna de un estado asociado.

\section{El aspecto económico de la cooperación en la ASEAN}

Las buenas calificaciones que Ia ASEAN ha obtenido hasta el momento en lo referente a su éxito político (la alta cuota de aceptación externa incluso de las posiciones comunes transmitidas hacia afuera en cuestiones conflictivas internacionales, el "lögro de un contexto de condiciones politicas de estabilidad y seguridad suficientes" 17 para los países miembros y para la región como un todo), no le son, en cambio, atribuidas unánimemente en cuanto a la cooperación económica. La Far Eastern Economic Review, altamente apreciada en el ámbito internacional, expresa una crítica muy extendida en su "Asia 1988 Yearbook"18: "Un factor fundamental que impide a los Seis alcanzar su meta ilusoria de integración económica es una falta de voluntad politica". No menos claramente se expresó Paul Leong, el representante del "Grupo de los Catorce" (una comisión de expertos creada en el año 1986 por la Cámara de Comercio e Industria de la ASEAN (ASEAN-GGI) con vistas a la cumbre de Manila), con motivo del Primer Congreso Económico de la ASEAN celébrado del 13 al 22 de marzo de 1987 en Kuala Lumpur. En su discurso inaugural" habló sin rodeos de un "record de negociaciones, exaspera-

\footnotetext{
${ }^{16} \mathrm{Cfr}$. Vitit Muntarbhorn, The Challenge of Law. Legal Gooperation among ASEAN Countries (Bangkok: Aksornsiam Press, 1986).

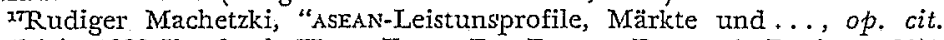

${ }^{18}$ Asia 1988 Yearbook. (Hong Kong: Far Eastern Economic Review, 1987), p. 74.

"Dato Paul Leong Khee Seong, "ASEAN: Choosing our Destinity", en Noordin Sopiel et al (eds.), $A S E A N$ at the Crossroad, op. cit., pp. 5-10; cit. p. 6.
} 
ciones y frustraciones prolongadas". Que tan severa sentencia se suscriba o no, depende de los criterios que se consideren.

Es cierto que las "principales áreas de cooperación económica de la ASEAN", determinadas ya en 1976 en la cumbre de Bali, no han satisfecho las expectativas que habían despertado. Para 1985, de todos los proyectos industriales comunes planificados en 1976, sólo se había puesto en marcha la fábrica de fertilizantes en base a nitrógeno en el norte de Sumatra (Indonesia), de modo tal que un reconocido especialista habla de una "experiencia por momentos traumática"20. Ni siquiera el Tratado de Preferencias Aduaneras de 1977, que en la actualidad incluye más de dieciocho mil ítemes, ha conducido a un incremento significativo del comercio dentro de la ASEAN, el cual comporta el $17 \%$ de las exportaciones de la región ${ }^{21}$. EI esquema de tratados de producción complementaria planeado en 1981 ofrece también dificultades. El desarrollo relacionado con el tratado sobre ASEAN Joint Ventures, firmado en 1983, continúa por el momento en pañales ${ }^{22}$.

Todas estas limitaciones pueden ser remitidas a algunos factores centrales: falta de claridad sobre Ia naturaleza y los objetivos de la cooperación económica, egoísmo nacional que se manifiesta precisamente en forma muy concreta en lo económico, la brecha entre los distintos niveles económicos y de desarrollo nacionales, la falta de una amplia complementaridad de las economías del caso, para las cuales las inversiones y la competencia del mercado tendientes a la especialización aparecen en forma todavía prematura.

El éxito económico de la Comunidad es, por lo tanto, hasta el momento menos ejemplar que el político. Por eso se ha hablado de un complicado "equilibrio entre el simbolismo y la capacidad de rendimiento". De igual modo se ha agregado que: "la politica puede prosperar en base al simbolismo perola economia no"23. De todas maneras, dicha fórmula ofrece motivo para no quedarse simplemente parados frente a la valoración provisional y ver también los aspectos positivos que la cooperación de la ASEAN ha traído para la economía hasta la actualidad. No puede considerarse como insignificante el hecho que, hoy en día, muchos de los altos líderes económicos de los países de la ASEAN se conozcan personalmente (justamente a raíz de encuentros en el marco de las actividades de la ASEAN), que en la economía de la región se vaya extendiendo la sen-

${ }^{\circ}$ Cfr. Chng Meng Kng, "ASEAN Economic Co-operation: The Current Status", en: Southeast Asia Affaires 1985 (Singapur: ISEAs, 1985), pp. 31-53; cit. p. 39.

${ }^{21}$ Südostasien Aktuell, julio de 1987, p. 312.

"Oskar Weggel, ASEAN. Regional und Aussenpolitik" en: Aus Politik and Zeitgeschichte 33/84, del 18 de agosto de 1984, pp. 17-32; cit. p. 32.

${ }^{23} \mathrm{C}$. P. F. Luhulima, "ASEAN institutions and modus operandi: Looking back and looking forward", en: Noordin Sopiee et al (eds.), Asean at the Crossroads, op. cit., pp. 161-181; cit. p. 178. 
sación de que se debería crecer juntos en modo más efectivo y múltiple, siguiendo la dinámica interna del comportamiento macroeconómico, y que, además, se esté dispuesto a trasladar este deseo operativa $y$ articuladamente al nivel político.

Es verdad que la cooperación política sectorial de los gobiernos de la ASEAN no se ve completada en forma inmediata por un crecimiento económico análogo. Sin embargo, por medio de contactos políticos, conversaciones, planes, etc. (quizás, o precisamente porque en una larga fase de ascenso estuvieron menos cargados de "costos" $y$, antes bien, proporcionaron experiencias en el trato mutuo), se han logrado las condiciones esenciales para aquella presión de aspiraciones que surge de la economía de los países de la ASEAN y que debe consignarse en la columna del haber del potencial económico de la Asociación.

Por un lado, la aceptación externa alcanzada por la Asociación en forma completa ha sido también una consecuencia de los esfuerzos transnacionales que marcharon por la vía de los órganos de representación de la economía de la ASEAN, sobre todo de la ASEAN-CCI, y que se siguen manteniendo. Por otro lado, las tasas de crecimiento de las economías nacionales de los países de la ASEAN, que están por encima del promedio internacional, apenas hubieran podido mantenerse a lo largo de tantos años si la comunidad entera no se hubiese abierto paso en la conciencia económica occidental como una de las regiones en crecimiento más significativas de nuestro tiempo. Los países de la ASEAN, y el calificativo de ASEAN es lo que que aquí cuenta, no hubieran llegado, con probabilidad cierta, al actual nivel de desarrollo económico sin la estrecha vinculación a los mercados occidentales y sin el (en términos de comparación internacional) sobreproporcional flujo de capitales norteamericanos, japoneses y europeo-occidentales, incluyendo sus posibilidades tecnológicas y de marketing correspondientes.

Las economías nacionales de Indonesia, Malasia, Tailandia, etc., exan atractivas sólo en parte en la consideración que los países industriales prestaron a la región. EI peso fundamental recayó en el proceso de crecimiento interestatal conjunto que se estaba llevando a cabo. Si, por ejemplo, en algún momento más de un tercio de las inversiones externas realizadas por el mundo occidental en los llamados países en desarrollo se volcaron a la región de los países miembros de la ASEAN, ello se produjo teniendo también en cuenta constelaciones venideras que no pueden ser separadas de la probabilidad del desarrollo futuro de la Comunidad. Por lo demás, el católogo de las medidas de cooperación económica en cuanto tales (preferencias aduaneras, proyectos industriales planificados según una especialización por países, joint ventures, complementaridad en 
la producción industrial, etc.) apunta claramente en la dirección correcta.

\section{Manifestaciones de crisis y propuestas de reforma}

La ASEAN ha dado buen resultado como un todo. Si, a pesar de ello, aparece la palabra "frustración" con respecto a lo alcanzado hasta aquí, ello no expresa una sensación de inutilidad (como, por ejemplo, en el caso de los esfuerzos de integración y cooperación de América Latina ${ }^{24}$ ), sino la desilusión de que todavía no se haya avanzado más. Quien hoy en día viaja por los países de la ASEAN siente la gravitación del pensamiento nacional estratégico y de desarrollo. A pesar de ello la ASEAN no es tenida como un compromiso adicional lujoso $u$ ob-truyente al que en última instancia se puede renunciar. Precisamente porque uno se hace pocas ilusiones acerca de la momentánea, aunque inevitable, tensión entre "nation building"25 $y$ el manejo regional futuro, siempre se ha visto la marcha del Sudeste Asiático hacia un regionalismo vital bajo el signo de un avance sucesivo y cauteloso. Lee Kuan Yew recordó esto una vez más en su discurso de apertura en Manila: "La ASEAN tiene un récord de veinte años de progreso y consolidación pacificos. No ha habido quiebras espectaculares pero si consultas permanentes y continuas que han llevado al consenso y a decisiones correctas"2o. En general hay mucho de cierto en ello. En los detalles, sin embargo, se vinculan con la ASEAN muchos problemas concretos, tales como elementos estructurales de perturbación, y también manifestaciones de crisis en las prácticas de cooperación llevadas a cabo hasta el momento. Pero sobre todo, después de veinte años de experiencias vividas de cooperación, se ve cada vez más la necesidad de reformas claramente orientadas.

Un elemento central de cohesión de la ASEAN en lo estratégico e ideológico consiste en rechazar los intentos comunistas en el interior $y$ en mantener distancia con respecto a las grandes potencias comunistas $y$ a las potencias comunistas regionales. Mientras que Ia defensa interna se lleva a cabo en forma generalmente exitosa, hacia afuera se aprecian manifestaciones de debilidad que llaman menos la atención allí donde la ASEAN actúa en forma colectiva. Las que se ven afectadas son las relaciones exteriores bilaterales de los gobiernos miembros. Por ejemplo, la distancia que con respecto a Vietnam trata de mantener Tailandia en su calidad de "Estado-

"Cfr. Manfred Mols (eds.), Integration und Kooperation in Lateinamerika (Padeborn: Schöningh, 1981); del mismo: El marco internacional de América Latina (Barcelona/Caracas: Alfa, 1985).

${ }^{2}$ "Cfr. Tengku Ahmad Rithauddeen, "Probleme und Aufgaben del Länder Südostasiens", Europa-Archiv, Nos. 4/41, 10 de marzo de 1986, pp. 145-153.

${ }^{2}$ Palabras de apertura del Primer Ministro Lee Kuan Yew, op. cit., p. 3. 
frente" tiene en la concepción de la política exterior de Yacarta un papel incomparablemente menor, porque Vietnam es considerado aquí como un contrapesó natural de la República Popular. Ghina. Tailandia cultiva una insignificante cooperación en política militar y de seguridad con China, que los otros miembros de la ASEAN toleran más que celebran. La cuestión de Camboya, que a veces ha sido vista (en forma exagerada según mi opinión) como el lazo unificante de la ASEAN en política exterior, ha conducido a la formación de subgrupos con simpatías y recelos diferentes ${ }^{27}$. En conjunto ha traído consigo el peligro de que potencias extranjeras, con sus propios intereses geoestratégicos, socaven la unidad de las posiciones de la ASEAN colocando a los gobiernos miembros de la Asociación frente a la tentación de perseguir el primado de una política de seguridad nacional por medio de "alianzas estratégicas".

Otro ejemplo reciente ha sido el intento de las Filipinas de alcanzar una regionalización de las bases militares americanas. El ministro del exterior Manglapus, quien trató de lograr la acéptación de esta idea haciendo una gira por los estados de la ASEAN, no pudo imponerse, a pesar de que todos los gobiernos habían aceptado a puertas cerradas la utilidad de la presencia americana en la región. E] ministro del exterior de Indonesia, Mochtar Kusumaatmadja, manifestó que la cuestión de las bases es un problema estrictamente bilateral entre las Filipinas y los Estados Unidos y "que lo mejor es que también pueda ser resuelto por Ias Filipinas y los Estados Unidos sin tener en cuenta el juicio de otros sobre las ventajas y desventajas de las bases'28.

Los cambios de la situación internacional, que no pueden ser calculados en detalle, contribuyen a la formación de:perspectivas tan diferentes. Cada aproximación americano-soviética más clara, cada cambio sustancial de las relaciones chino-soviéticas, y también el comportamiento de los Estados Unidos, de la Unión Soviética, de China o de Japón (que no pueden preverse en lo que hace a la política mundial y ni siquiera. en relación con el Sudeste Asiático), hacen pensar en constelaciones internacionales cambiantes que traen consigo "distintas inseguridades" 29 para los miembros de la Asociación. La reacción presumible de intentạ influir sobre los presuntos cambios de contexto y/o por lo menos amortiguarlos respondiendo con posiciones conjuntas de la ASEAN encuentra (ipor el momento?) su límite en el hecho de que las prioridades de la poli-

${ }^{z}$ Cfr. Lau Teik Soon, "Shifting Alignments in Regional Politics", en: Southeast Asian Affaires 1986 (Singapur: IsEAs, 1986), pp. 3-24.

${ }^{3}$ Comunicado de prensa de la Embajada de la República de Indonesia del 19 de noviembre de 1987, (No 20 / pen XI /87).

${ }^{2} \mathrm{CFr}$. Kernial S. Sandhu, "The Potential of ASEAN", en: Yoshiyuki Hagiwara, Asia in the 1980s: Interdependence, Peace, Development (Tokio, 1982), p. 111117; cit. p. 113. 
tica exterior, de seguridad y de bienestar diferentes aquí y allí tienen que ser respetadas. Ningún cambio en esta cuestion ha sido realizado por los jefes de Estado y de gobierno reunidos en Manila.

En la ASEAN no se trata primordialmente de una armonización regional sino de alcanzar, por medio de la cooperación, el máximo posible de éxitos en ámbitos políticos exactamente definidos "con costos y riesgos aceptables" (Sutresna). Esto parece altamente plausible en vista de las perturbaciones históricas de la región. ¿̨Se sabe con tanta exactitud; sin embargo, en las capitales de los paises de $I_{a}$ ASEAN, por dónde pasa el corte entre ventajas, costos y riesgos nacionales y comunitarios? Esta pregunta vale también con relación a la vinculación externa de las economías de los países de ía ASEAN. Los éxitos de industrialización y desarrollo de la subregión se han pagado al precio de una fuerte dependencia de tecnologías y know how extranjeros. Las multinacionales y financieras japonesas, europeas y norteamericanas ganan año a año mayor influencia y tornan dificil el control político nacional de la economfa nativa en razón, entre otras cosas, de un vacío sostenido de inversiones que sólo puede ser financiado desde el exterior. También el choque con el límite de la capacidad de absorción de los mercados en los países socios en el diálogo (proteccionismo) indica que el éxito económico no se obtiene por si solo. Entretanto, las tasas de crecimiento económico de Corea del Sur, Taiwán y Hong Kong han superado en mucho a las de Ios pafses de Ia ASEAN. ¿No había residido, sin embargo, la atracción expresada en la ya descrita aceptación externa de la ASEAN, entre otras cosas, en el hecho que se la considera como una de las últimas regiones de crecimiento promisorio e inagotable de la tierra?

Los éxitos de la ASEAN están vinculados, tanto hacia el interior como hacia el exterior, con la imagen de una estabilidad política extraordinaria. Dejando de lado que en tales apreciaciones normalmente se confunde la continuidad de la conducción política con el fenómeno incomparablemente más complicado de la estabilidad, hoy en día se discuten con mayor claridad que antes los elementos regionales de peligro para la dominación política: tensiones étnicas, irrupciones de fundamentalismo religioso, movimientos de oposición, disminución tendencial del conformismo de los sindicatos, manifestaciones de independencia de los militares, fallas en el funcionamiento de la administración; corrupción, evidentes deformaciones de la distribución, etc. Además la continuidad de la conducción política en Malasia, y posiblemente también en Tailandia, se ve amenazada por tensiones internas. En Singapur y en Indonesia se prepara un cambio político generacional. Las Filipinas demuestran lo que un relevo del poder político significa en pafses con estrecha base de legitimación. 
Evidentemente tales imponderables no vuelven en modo alguno obsoletos los pensamientos de reforma que ya se han puesto en marcha. Los mismos fueron propuestos en forma, si no me equivoco, especialmente exacta y atendiendo a detalles precisos en el Primer Congreso Económíico de la ASEAN en Kuala Lumpur bajo el patrocinio del "Grupo de los Catorce"30 y por ISEAs en Singapur". En ninguno de ambos casos se pasaron por alto las anteriores propuestas de reforma e innovación, como por ejemplo la del Task Force Report presentado en $1983^{32}$. La Conferencia de Kuala Lumpur fue una reunión multinacional a la que llegaron importantes documento: provenientes de los restantes países miembros de la ASEAN.

En esta nueva discusión de reformas se perfilan tres sugerencias y propuestas principales:

-Ya sería hora de que la Comunidad comenzara a "fijarse objetivos más ambiciosos" (Kintanar). Si las prioridades nacionales han de seguir siendo respetadas, la forma de cooperación pragmático-explorativa llevada a cabo hasta el momento debería entonces dejar paso a una clara fijación de objetivos. La palabra "integración" no debería ser relegada a una zona tabú, sobre todo si queda abierta a distintas interpretaciones de contenido. El marco jurídico laxo no sería en caso alguno suficiente para orientar la dinámica del futuro desarrollo de la ASEAN.

- También se tendría a disposición la estructura organizativa. La conferencia de jefes de Estado y de Gobierno habría de reunirse anualmente y cumplir realmente con su función de supremo órgano de decisión de la Comunidad. Las reuniones de ministros de economía, del exterior y otros, que no de jure pero sí de facto, a veces compiten y se obstaculizan mutuamente, deberian ser reemplazadas por un consejo de ministros. El "Comité Permanente" rotativo habría de ser sustituido por un "Comité de Representantes Permanentes" que se establecería en Yakarta. La Secretaría de Ia ASEAN en la misma ciudad debería ser dotada de competencias mucho más amplias y recursos suficientes. También las vinculaciones entre la ASEAN-CGI, las restantes Organizaciones No Gubernamentales (ONGs) y los gremios oficiales de la Asociación serían entonces llevadas a cabo por la Secretaría, fortalecida de ese modo en sus competencias y capacidades.

${ }^{30} \mathrm{Cfr}$. Ia obra colectiva en nota 7 y $A S E A N_{i}$. The Way Forward. The Report of the Group of Fourteen on ASEAN Economic Cooperation and Integration (Kuala-Lumpur: Institut of Strategic and International Studies; 1987).

"Cfr. sobre todo: ISEAS, "ASEAN, The Taks Ahead", ASEAN Economic Bulletin, Vol. 2, No 1, 985, pp. 1-12.

"Detalles en: Eberhard Knappe, "The Task Force Report - Zusamennsetzung und Kommentierung", en: Helger Hanel et al, Bericht über das Projekt ASEAN. Ziele, Formen, Probleme und Konsequenzen regionaler Zusammenarbeit in Südostasien', Berlin, mimeo, 1987, pp. 79-154. 
$\therefore$-Sería imprescindible una cooperación económica mucho más estrecha, también transformada conceptualmente, que sea liberada de los tradicionales impedimentos de la supervisión administrativa de los gobiernos nacionales. Bajo el estímulo proporcionado por aIgunas manifestaciones ${ }^{\mathbf{3}}$ de políticos líderes de distintos países miemJros, que son capaces de imaginarse una consolidación de la cooperación económica realizada a través de etapas sucesivas, ha surgido del ISEAS, conducido por Hans Ghristoph Rieger, la propuesta de establecer en la ASEAN un sistema de comercio sobre la base de la "Construcción 4+2": Indonesia, Malasia, Filipinas y Tailandia deberían formar una unión aduanera $\mathrm{y}$ junto con los países miembros de Singapur y Brunei, constituir una zona de libre comercio.

Además de estos tres paquetes fundamentales de reformas (fijación más. precisa de los objetivos, aumento de la efectividad de la organización y nueva concepción del marco operativo de la cooperación económica) hay una serie de otras propuestas que apuntan a una intensificación de la cooperación en lo que se refiere a los intereses industriales, a un mejoramiento de los ámbitos fiscal, monetario y bancario y a un reforzamiento de los intercambios en educación, investigación, comunicación etc., que ya han sido puestos en marcha.

La lógica de las propuestas se evidencia por sí misma. Se apela a la voluntad política de los responsables de decisiones nacionales para enfrentar la dinámica inhibitoria que ha venido desarrollándose. Esto implicaría un crecimiento del mercado libre, es decir, del libre juego de fuerzas económicas y, también, un aumento en la cons:deräción que se tiene de las fuerzas configurantes de los grupos sociales pensados en la perspectiva de la ASEAN.

\section{Nuevamente sobre la Cumbre de Manila}

La cumbre de Manila se demostró pobre en comparación con este complejo paquete de reformas. Seguramente ha contribuido a ello la incertidumbre acerca de su realización mencionada al comienzo, que en la práctica organizativa condujo a que la 'Conferencia haya sido celebrada en forma abreviada. El premier de Singapur, Lee Kuan Yew, se ha referido varias veces (con propiedad a mi entender) a otros tiempos ${ }^{34}$, diciendo que la ASEAN ha surgido y se ha decarrollado en una constelación histórica muy especial que ha dejado huellas en las experiencias políticas de sus líderes. "Para aquellos ahora' en la mitad de su vida, cuya mayor experiencia fue la lucha por preservar la independencia y por mantener la unidad nacional

\footnotetext{
"Cfr. Hans Christoph. Rieger, "The Market Economies of Southeast Asia in 1985: ASEAN Pays the Price", en Southeast Asian Affairs 1986 (Singapur: IsEAs, 1986), pp. 12-30; cit. p. 27.

Palabras de apertura..., op, cit., p. 5.
} 
frente a la insurrección comunista armada y frente a las tendencias divisorias de las distintas razas, lenguas y religiones". Continúa: "Nuestra juventud no recuerda los conflictos pasados. Cionsidera los últimos veinte años de paz y prosperidad como el curso natural de los sucesos". También -en calidad de comentarista científico- se podría preguntar. si sería acaso insensato ser consecuentemente cuidadosos con compromisos que, quizás, una próxima generación de dirigentes futuros juzgue en forma muy distinta.

A pesar de que en Manila ha quedado mucho pendiente, pueden reconocerse, sin embargo, los puntos débiles y los centros de gravedad de la discusión.

Primero, se ha tomado una serie de medidas para intensificar la cooperación económica. "Entre ellas se hallan en primer plano la eliminación de las barreras aduaneras y el fomento de las inversiones privadas"35. Norbert Wagner, a quien pertenece este resumen, comenta así al respecto: "Las medidas tomadas ahora se concentran en mejorar el marco de condiciones para la cooperación en el sector privado. Eso indica que los gobiernos de la ASEAN han comprendido ahora que los grandes proyectos y la cooperación ordenada 'desde arriba' no tienen éxito".

El segundo elemento que hay que tener en cuenta aquí es que los jefes de gobierno de la ASEAN se han dedicado por primera vez desde la constitución de la Comunidad a un fundamental intercambio de opiniones sobre las cuestiones de política de seguridad. Ya se ha indicado más arriba que aquí existen divergencias, en parte de naturaleza fundamental. Además de los ya anunciados puntos de controversia, Indonesia hubiera querido, por ejemplo, concretizar el concepto zopfan. Indonesia y Malasia también hubieran deseado llegar a un tratado sobre una zona libre de armamentos nucleares. Los demás gobiernos, en cambio, opinaron que zopfan no podrfa realizarse en forma inmediata a causa de la cuestión no resuelta de Camboya y que entonces debería permanecer como un concepto formativo a largo plazo. En lo que hace a la zona libre de armamentos nucleares se pronunciaron de todos modos por derivar la cuestión a una comisión evaluadora.

En tercer lugar ha de mencionarse el documento de cierre: la Declaración de Manila de 1987. En ella no puede pasarse por alto la absolutamente decisiva declaración de la voluntad de continuar y desarrollar la Asociación, ni su no menos decisiva fundamentación: "El regionalismo de la ASEAN, fundado en la cohesión económica, politica y cultural, es más vital que nunca para el futuro de la $A S E A N " 36$. A continuación se subraya la intención: "La $A S E A N$ ha

${ }^{\times C f r}$. el trabajo cit. en nota 2.

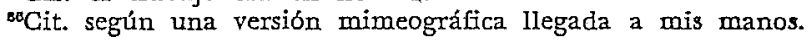


de perseguir la solidaridad y la cooperación regionales bajo cualquier circunstancia...". La fuerte apelación al aspecto político de la cooperación aparece nuevamente donde se enumeran los campos de cooperación de la ASEAN: la "cooperación política" se coloca inequívocamente en primer lugar. EI tramo que en este sentido ha recorrido la Comunidad en los veinte años de su existencia se aprecia en que en el documento fundacional de Bangkok el aspecto político aparece sólo en forma implícita.

En detalle, muchos puntos quedan abiertos y son más una declaración de intenciones que resoluciones aun cuando, además de Ia declaración de Manila, existen algunos tratados complementarios de los ministros. Sin embargo tno ha conseguido la ASEAN sus verdaderos éxitos del pasado renunciando conscientemente a enormes convenciones y tratados jurídicos imposibles de ser cumplidos en forma realista y avanzando flexible y pragmáticamente por medio de pasos bien meditados y viables?

No se puede reflexionar sobre la ASEAN sin recordar un punto sensible de la cosmovisión de los estratos dirigentes del Sudeste Asiático: la primacía indiscutida hasta el momento del concepto de "nation building" aplicado a cada uno de los estados, prioridad que tampoco se ve afectada en sustancia por el ajuste de "resilience" nacional y regional definido dialécticamente. De todos modos los propagadores de otras asociaciones regionales fuera de Europa pueden envidiar a Ios reformadores de la ASEAN y sus problemas. Políticamente la ASEAN se enfrenta al dilema del rey: se ve sobrepasada por sus propios éxitos. Por eso no es ninguna casualidad que hoy en día se observen y discutan con mayor atención que antes las experiencias de integración de terceros: de la Comunidad Europea, de la AELC y del Pacto Andino.

- Paul Leong ha señalado en el Primer Congreso Económico de la ASEAN en Kuala Lumpur un criterio para el desarrollo futuro de la Comunidad, que es ambicioso y apunta más allá del cambio de siglo: "Sólo algunas sociedades tendrán éxito en su intento de lograr el bienestar entre las naciones: las que tengan tenacidad resistente y firmeza, las que tengan la visión, la resolución y el coraje de enfrentar los desafios y determinar su futuro. Las sociedades menos dotadas se quedan en el camino. La historia ha demostrado esto repetidas veces y nos ha dado suficientes ejemplos de que el futuro no es nuestro, a menos de que lo elijamos nosotros mismos"37.

${ }^{27}$ Cit. en nota 7, p. 5 . 